Article

\title{
Synthesis of a New Imidazole Amino Acid Ionic Liquid Polymer and Selective Adsorption Performance for Tea Polyphenols
}

\author{
Yingjie Luo, Xiaoxia Huang, Shun Yao $₫$, Lincai Peng, Fulin Li and Hang Song * \\ Department of Pharmaceutical and Biological Engineering, School of Chemical Engineering, Sichuan University, \\ Chengdu 610000, China; luoyingjie1115@163.com (Y.L.); 15528091852@163.com (X.H.); cusack@scu.edu.cn (S.Y.); \\ wacord@163.com (L.P.); lifulin2017@163.com (F.L.) \\ * Correspondence: hangsong@scu.edu.cn; Tel.: +86-28-8540-5221; Fax: +86-28-8540-3397
}

Received: 19 August 2020; Accepted: 21 September 2020; Published: 23 September 2020

check for updates

\begin{abstract}
A series of imidazolium ionic liquid monomers with L-Proline anions (ViImCn-L-Pro and (ViIm)2Cn(L-Pro)2) were firstly synthesized, after which new copolymer materials were prepared by polymerization of the ionic liquid monomers with $\mathrm{N}, \mathrm{N}^{\prime}$-methylene diacrylamide (MBA). Polymerization conditions, including the ratio of Ils(ViImC $\mathrm{C}_{\mathrm{n}}-\mathrm{L}-\mathrm{Pro}$ or $\left.(\mathrm{ViIm})_{2} \mathrm{C}_{\mathrm{n}}(\mathrm{L}-\mathrm{Pro})_{2}\right)$ and MBA, solvent, ionic liquids and initiator's amount, were investigated and found to have an important effect on the adsorption capacity. Polymerization conditions were shown to have more significant impacts on adsorption capacities in the following order: the ratio of Ils and MBA > the amount of initiator $>$ ionic liquids $>$ solvent. The polymers were characterized by IR, EA, SEM, particle size distribution and TG. One of the polymers exhibited the highest selective adsorption capacity of tea polyphenols $(521 \mathrm{mg} / \mathrm{g})$. which was significantly higher than other adsorption media. The absorbed tea polyphenols could be desorbed readily with $2 \%$ hydrochloric acid methanol solution as eluent. The polymer material could maintain a higher adsorption capacity after four reuses. Based on this polymer, a new method for the efficient separation of tea polyphenols from tea water could be developed.
\end{abstract}

Keywords: ionic liquid polymer; imidazole; amino acid; adsorption; tea polyphenols

\section{Introduction}

Ionic liquids (ILs) are a developing material and have been widely applied in extraction, adsorption and catalysis. Some dicationic ILs exhibit, in their structures, a higher adsorption capacity [1,2]. Poly(ionic liquid)s combine the characteristic of ILs with polymers and have many excellent properties, such as a better mechanical stability, ionic conductivity, processability, durability, chemical compatibility and controllability [3,4]. Poly(ionic liquid)s have special structures and could therefore have multiple functions [5-8]. In recent years, polyionic liquids have attracted great attention in the fields of materials, separation and purification, electrochemistry and catalysts [9-12]. The safety of imidazole-based ILs has been demonstrated in the past years [13]. Some natural amino acid-based imidazole ILs have a good biocompatibility, nontoxicity and other characteristics, and thus a better safety [14-18].

Tea polyphenols (TPs), including Epigallocatechin gallate (EGCG), Epicatechin (EC) and Epigallocatechin gallate (ECG), constitute a high content in tea, accounting for $15-30 \%$ of the dry weight of tea. TPs have multiple biological activities, such as oxidation resistance, preventing cardiovascular diseases or relieving atherosclerosis and arrhythmia [19,20]. TPs are difficult to obtain by chemical synthesis due to their complex chemical structures and poor chemical stability. Therefore, TP product is made mainly from tea, and its production involves a complex process that is composed of a series 
of processes. The TPs and other substances are first extracted from tea raw materials and then purified. To obtain high-purity TPs, a series of different separation methods and equipment have to be applied to remove various other compounds, such as pectin, polysaccharide, theophylline, caffeine and pigment [21].

Resin adsorption is one of purification methods used for TPs. For example, Dong and others [22] chemically synthesized adsorption resin NKA-2 as an adsorbent, and $80 \%$ ethanol was used as effluent solvent. However, the investment and operation costs are relatively high. In particular, the adsorption process has to use a large amount of volatile organic solvents, so the effects on ecological environmental protection and safety are not satisfactory. In recent years, several new methods for the separation and purification of TPs have been reported. Luo and others [23] prepared a porous starch that exhibited a selective adsorption performance on TPs with an adsorption capacity of $25.72 \mathrm{mg} / \mathrm{g}$. It has some advantages, such as less organic solvent, mild adsorption conditions and good adsorption selectivity. However, a low adsorption capacity would lead to very large amount of adsorbent being required, which is difficult to realize in industrial applications. A good adsorption selectivity and high adsorption capacity are quite challenging and very significant.

In our previous study, ionic liquids were revealed to show a good selective separation of tea polyphenols and caffeine from tea leaves [24]. The adsorption capacity reached $162 \mathrm{mg} / \mathrm{g}$. The adsorption active sites of the ILs are likely partially shielded after immobilization, which made it difficult to achieve a great adsorption capacity. ILs' polymerization can replace the immobilization and ensure a high availability of active adsorption sites in the adsorption process.

In this work, a series of imidazole-type bicationic amino acid-based ILs ((ViIm) ${ }_{2} \mathrm{C}_{\mathrm{n}}$ (L-Pro) ${ }_{2}$; $\mathrm{n}=3-6$ ) were synthesized, and then new ILs polymers were prepared with the ILs and $\mathrm{N}, \mathrm{N}^{\prime}$-methylene diacrylamide (MBA). In addition to the characterization of the polymers, their adsorption capacities and selectivities for TPs were tested.

\section{Experimental Section}

\subsection{Materials and Reagents}

$\mathrm{N}$-vinyl imidazole $(>98.0 \%)$, L-proline and $\mathrm{N}, \mathrm{N}$-methylene bisacrylamide were purchased from Titan Scientific Co., Ltd. (Shanghai, China). 1-bromopropane $(>98.0 \%)$, 1-bromobutane, 1-bromopentane, 1-bromohexane, 1,3-dibromopropane, 1,4-dibromobutane, 1,5-dibromopentane, 1,6-dibromohexane, strong alkalinityanion-exchange resin, sodium acetate, potassium peroxodisulfate, concentrated hydrochloric acid $(36 \%$,), ethyl alcohol and methyl alcohol were supplied by Kelong Chemical Co., Ltd. (Chengdu, China). Sodium citrate, sodium chloride, ammonium sulfate, theophylline and tea polyphenol were obtained from Aladdin Biochemical Technology Co., Ltd. (Shanghai, China). All reagents were AR grade with 99\% purity, except indicated otherwise.

\subsection{Apparatus}

Element analysis data were obtained from an EA3000 elemental analyzer (EuroVector Instruments and Software, Milan, Italy). An Infrared L1600300 Spectroscopy (PerkinElmer, Fremont, CA, USA) was used to record Fourier transform infrared spectra (FT-IR) in the range of $4000-400 \mathrm{~cm}^{-1}$ with $\mathrm{KBr}$ pellets. The morphology was observed with JSM-7001F scanning electron microscopy (JEOL Co., Ltd., Tokyo, Japan). The thermogravimetric analysis (TGA) was performed on Microcomputer differential thermobalance HTG (Hengjiu scientific instrument, Beijing, China) with a heating rate of $10^{\circ} \mathrm{C} \mathrm{min}-1$ from 30 to $800{ }^{\circ} \mathrm{C}$ under nitrogen. The particle size distribution was measured by a JL-1198 laser particle size analyzer equipped with a nonuniform cross arranged three-dimensional sector matrix detector in the range of 0.1-600 $\mu \mathrm{m}$ and an He-Ne gas laser (Run Technology Co. Ltd., Henan, China). The total content of tea polyphenols was determined by a UV-2800 spectrometer (Hengping scientific instrument, Shanghai, China). The quantitative analysis was performed on a high-performance liquid chromatographic system equipped with an LC-20AT pump, an SPD-M20A photodiode array detector 
and a Class-VP workstation (Shimadzu, Kyoto, Japan). The analytical column was a Symmetry C18 column $(4.6 \times 250 \mathrm{~mm}, 5 \mu \mathrm{m}$, Waters, MA, USA). The analytical conditions for the actual extracts were as follows: the mobile phase consisted of methanol-water-methanoic acid (20:79.4:0.6, v/v/v); the flow rate was $1 \mathrm{~mL} \mathrm{~min}{ }^{-1}$; the column temperature was maintained at $25^{\circ} \mathrm{C}$; the detection wavelength was set at $278 \mathrm{~nm}$, and a $10-\mu \mathrm{L}$ aliquot of sample solution was injected and analyzed.

\subsection{Synthesis of Imidazole Ionic Liquid Polymers}

\subsubsection{Synthesis of ILs monomer ViImCn-L-Pro and (ViIm)2Cn(L-Pro)2}

The synthetic routes of (Vilm)2Cn(L-Pro)2 are shown in Figure 1. $\mathrm{Br}\left(\mathrm{CH}_{2}\right)_{n} \mathrm{Br}$ and N-Vinylimidazole in a molar ratio of 1:2 were mixed in an appropriate amount of solvent acetonitrile. The mixture was kept at reflux at $82{ }^{\circ} \mathrm{C}$ while stirring for $24 \mathrm{~h}$, and then light yellow liquid was obtained. After the solvent was removed, the intermediate product was washed with ethylacetate and acetone in a solid-liquid ratio of 1:10 1-2 times in sequence and was then dried under vacuum for $24 \mathrm{~h}$. An aqueous solution of $(\mathrm{ViIm})_{2} \mathrm{C}_{\mathrm{n}}(\mathrm{Br})_{2}$ $(\mathrm{n}=3,4,5,6)$ was made to prepare $(\mathrm{ViIm})_{2} \mathrm{C}_{\mathrm{n}} \mathrm{OH}$, with an exchange reaction in styrene $201 \times 7$ strongly basic anion exchange resin. HPLC was used for monitoring the progress. In the last step, slightly excessive L-proline was added into (ViIm) ${ }_{2} \mathrm{C}_{\mathrm{n}} \mathrm{OH}$ solution and stirred at room temperature for $24 \mathrm{~h}$. After the reaction, the solvent was removed, and a burgundy viscous oily substance, the target product of ionic liquid (Vilm) ${ }_{2} \mathrm{C}_{\mathrm{n}}(\mathrm{L}-\mathrm{Pro})_{2}$, was obtained. The product was placed in a low-temperature dry environment for later use.

a
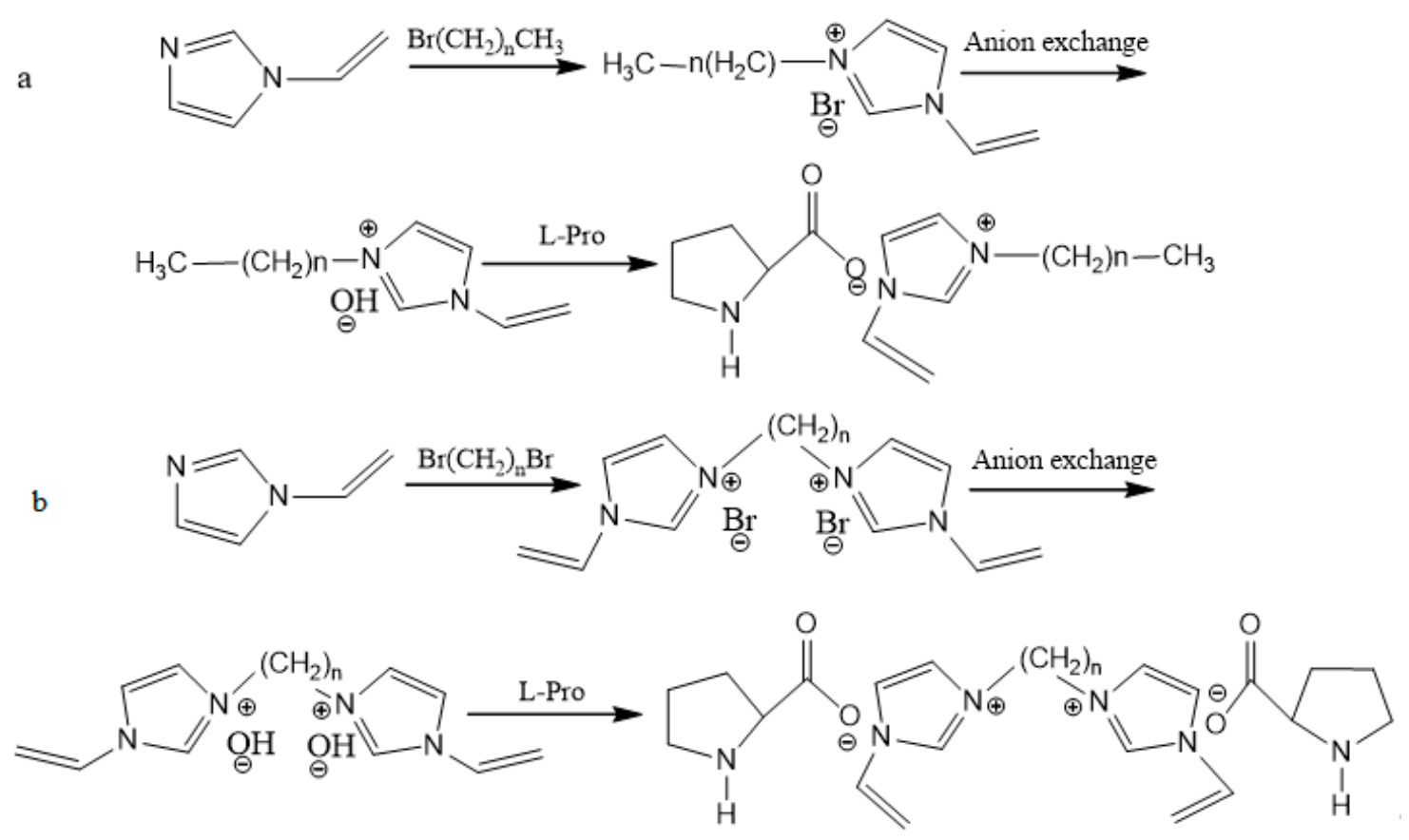

Figure 1. The synthesis of (a) ViImCn-L-Pro and (b) (ViIm) ${ }_{2} \mathrm{C}_{\mathrm{n}}$ (L-Pro $)_{2}$.

The synthesis methods of ViIm $\mathrm{C}_{\mathrm{n}}$-L-Pro were similar to those of $(\mathrm{ViIm})_{2} \mathrm{C}_{\mathrm{n}}(\mathrm{L}-\mathrm{Pro})_{2}$. The synthesis routes are shown in Figure 1. The structural characterization of the ionic liquid is shown in the Supplementary Materials.

\subsubsection{Synthesis of Imidazole Ionic Liquid Copolymers}

ILs as monomers were directly polymerized with cross-linking agent MBA to form polymer. Different molar ratios $(0: 1,0.25: 1,0.5: 1,0.6: 1,0.75: 1,1: 1)$ of $(V i I m)_{2} C_{n}(\mathrm{~L}-\mathrm{Pro})_{2}(\mathrm{n}=3,4,5,6)$ to MBA were investigated to prepare poly(ionic liquid)s. The six polymers were labeled Pol 0, Pol a, Pol b, Pol c, Pol d, Pol e. The dosage of $\mathrm{K}_{2} \mathrm{~S}_{2} \mathrm{O}_{4}$ was 3.5\% (the ratio of the initiator addition to the total 
mass of the reaction raw materials) as the initiator. The solvent (water, methanol, ethanol, etc.) was ultrasonically vibrated to uniformly disperse the initiator. Inert gas $\mathrm{N}_{2}$ was added to exhaust oxygen to carry out the reaction at $50{ }^{\circ} \mathrm{C}$. After the reaction, it was washed twice with pure water and dried for $48 \mathrm{~h}$. The polymerization route is shown in Figure 2.

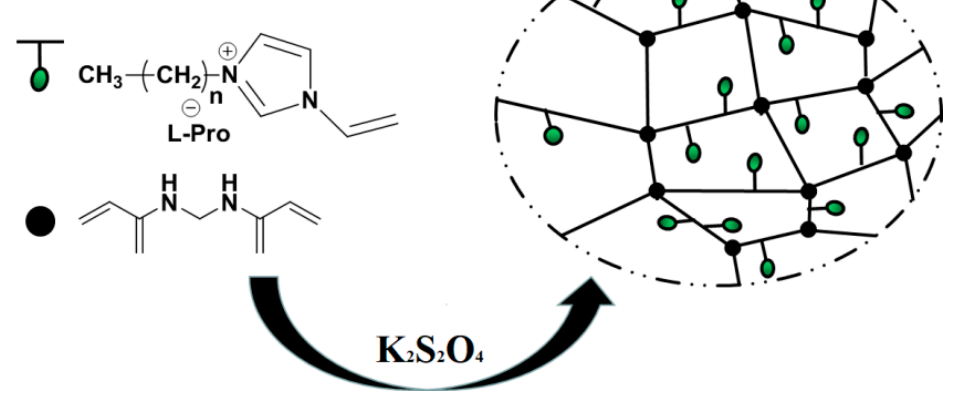

Figure 2. Synthesis routes of imidazole IL copolymers.

\subsection{Structural Characterization of Ionic Liquid Polymer}

The poly(ionic liquid)s were characterized with an infrared spectrometer (FT-IR), thermogravimetric analysis (TG), elemental analysis (EA), scanning electron microscope (SEM) and particle size distribution. The infrared spectrum (FT-IR) was measured by transmission method (KBr tableting) on a Perkin Elmer Fourier infrared spectrometer. The thermogravimetric analyzer (TG) was measured by using a microcomputer differential thermal balance. The elemental analysis (EA) was detected by using a EURO EA3000 type element analyzer. The determined elements included $\mathrm{C}, \mathrm{H}, \mathrm{N}$ and $\mathrm{O}$. The content of $\mathrm{IL}$ in the polymer was calculated by the content of $\mathrm{N}, \mathrm{O}$, and a scanning electron microscope (SEM) was used to observe the surface morphology of the materials. The particle size distribution was measured on a JL-1198 nanolaser particle size analyzer.

\subsection{Determination of Polymers' Yield and Mass Ratio of Ionic Liquids in Polymers}

In this experiment, a certain amount $\mathrm{ViImC}_{3}$-L-Pro was employed as the polymerization monomer, which was directly polymerized with cross-linking agent MBA to form polymer. $\mathrm{K}_{2} \mathrm{~S}_{2} \mathrm{O}_{4}$ was selected as the initiator and accounted for 3\% of the total mass of the reaction raw materials. The solvent was water. When this reaction was finished, the polymer was dried for a certain time and weighed. Elemental analysis was used to analyze the content of $\mathrm{N}, \mathrm{O}$ and to calculate the mass ratio of ILs. The yield was calculated according to the mass of the initial reactants and product. These equations were (1) and (2):

$$
\begin{aligned}
\text { mass ratio } & =15.75 \times\left(1.14 \mathrm{P}_{\mathrm{N}}-\mathrm{P}_{\mathrm{O}}\right) \\
\text { yield } & =\frac{\mathrm{m}_{\text {polymer }}}{\mathrm{m}_{\mathrm{MBA}}+\mathrm{m}_{\mathrm{ILs}}}
\end{aligned}
$$

$\mathrm{P}_{\mathrm{N}}$ is the content of $\mathrm{N}$ in the polymer. $\mathrm{P}_{\mathrm{O}}$ is the content of $\mathrm{O}$ in the polymer. $\mathrm{m}_{\text {Polymers }}(\mathrm{g})$ is the mass of the polymer. $\mathrm{m}_{\mathrm{MBA}}(\mathrm{g})$ is the quality of the initial response of the MBA. $\mathrm{m}_{\mathrm{Ils}}(\mathrm{g})$ is the quality of the initial response of the Ils.

\subsection{Optimization of Polymer Synthesis Conditions}

\subsubsection{Influence of the Ratio of Ionic Liquid and Crosslinking Agent MBA on Adsorption}

$0.6 \mathrm{~g}$ of cross-linking agent (MBA) was added in $30 \mathrm{~mL}$ distilled water to form a solution. Then, a certain amount of IL (ViImC $\mathrm{C}_{3}$-L-Pro) was dissolved in the solution with ultrasound assistance and was left standing for three hours. An appropriate amount of initiator $\mathrm{K}_{2} \mathrm{~S}_{2} \mathrm{O}_{4}$ was dissolved in the 
solution with ultrasound assistance. Nitrogen gas was introduced for $15 \mathrm{~min}$ to remove oxygen, the solution was placed at $50{ }^{\circ} \mathrm{C}$ for $6 \mathrm{~h}$, and a light yellow polymer was obtained. It was washed twice with pure water and dried under vacuum for $48 \mathrm{~h}$. A polymer of 200-350 mesh was prepared by grinding and sieving it for the adsorption test.

\subsubsection{Influence of Solvents on Adsorption}

Water, methanol and ethanol were used, respectively, as a polar solvent to investigate their influence. $\mathrm{K}_{2} \mathrm{~S}_{2} \mathrm{O}_{4}$ was used as the initiator when water was used as a solvent. However, AIBN was used as the initiator when methanol or ethanol were used as a solvent, due to the lower solubility of $\mathrm{K}_{2} \mathrm{~S}_{2} \mathrm{O}_{4}$ in methanol or ethanol. The polymerization was carried out at a ratio of $n(I L): n(M B A)=1: 3$.

\subsubsection{Influence of Ionic Liquids on Adsorption}

Under the ratio of $\mathrm{n}(\mathrm{IL}): \mathrm{n}(\mathrm{MBA})=1: 3,(\mathrm{ViIm})_{2} \mathrm{Cn}(\mathrm{L}-\mathrm{Pro})_{2}(\mathrm{n}=3,4,5,6)$ and ViImCn-L-Pro(n = 3, $4,5,6)$ were taken as monomers, respectively, in the case of a water solvent and a $\mathrm{K}_{2} \mathrm{~S}_{2} \mathrm{O}_{4}$ initiator in order to synthesize the polymers.

\subsubsection{Influence of Initiator Amount on Adsorption}

$(\mathrm{ViIm})_{2} \mathrm{C}_{6}$ (L-Pro) $)_{2}$ was used as a monomer. Under the optimal ratio of $\mathrm{n}(\mathrm{IL}) \mathrm{n}(\mathrm{MBA})$, a total mass of the initiator $\mathrm{K}_{2} \mathrm{~S}_{2} \mathrm{O}_{4}$ of $2.5 \%, 3.5 \%, 4.5 \%, 6.0 \%, 9.0 \%$ and $12 \%$ was selected, respectively, to synthesize the polymer in the water solvent.

\subsection{Adsorption Test of TPs}

\subsubsection{Establishment of Determination Method for TPs}

The total TP content in the adsorption experiment was analyzed by using the area normalization method by comparing the spectra with the standard tea polyphenols and theophylline that were purchased.

\subsubsection{Adsorption Performance Test}

$40.0 \mathrm{mg}$ polymer (mesh size 200-350) was mixed with a $20.0 \mathrm{~mL}$ solution of $1 \mathrm{mg} / \mathrm{mL} \mathrm{TP}$ concentration, and the mixture was placed in a vibrator $(200 \mathrm{rpm})$ at a certain temperature for the TPs' adsorption. The adsorption conditions were investigated under the following conditions: the solid-liquid ratio was 60:20; the adsorption temperature was $25^{\circ} \mathrm{C}$; the adsorption time was $6 \mathrm{~h}$; and the initial concentration range was between $0.5-3.5 \mathrm{mg} / \mathrm{mL}$. After $360 \mathrm{~min}, 1.00 \mathrm{~mL}$ of the supernatant sample was taken and analyzed at $273 \mathrm{~nm}$ by HPLC to determine each compound's content with an area normalization. Then, the TPs' adsorption capacity $q_{\mathrm{e}}(\mathrm{mg} / \mathrm{g})$ and the adsorption selectivity $S$ of the adsorbent could be calculated according to Equations (3) and (4):

$$
\begin{gathered}
q_{\mathrm{e}}=\text { mass }(\mathrm{mg}) \text { of absorbed TPs/mass }(\mathrm{g}) \text { of absorbent } \\
S=\text { mass }(\mathrm{mg}) \text { of absorbed TPs/mass }(\mathrm{mg}) \text { of absorbed theophyline }
\end{gathered}
$$

\section{Results and Discussion}

\subsection{Characterization of Ionic Liquid Polymers}

\subsubsection{Infrared Spectroscopic Analysis}

The infrared spectrum characterization results of IL (ViImC 3 -L-Pro), Pol 0 (MBA polymer only) and Pol c ((VilmC 3 -L-Pro):MBA $=3: 5)$ are shown in Figure 3. The main characteristic peaks of Pol 0 are the bending vibration absorption peak of $\mathrm{C}=\mathrm{O}$ at $1651 \mathrm{~cm}^{-1}$ and the strong absorption peak of the $\mathrm{N}-\mathrm{H}$ 
bending vibration at $1528 \mathrm{~cm}^{-1}$ (unique to the N-H anti conformation) [25]. Similarly, corresponding absorption peaks could also be found at the corresponding positions in Pol c, which meant that MBA components participated in the formation of Pol c. The absorption peak of Pol c at $3429 \mathrm{~cm}^{-1}$ was significantly enhanced when comparing the infrared signals of Pol 0 and ILs. It was possible that the water absorption of amino acid-based ILs itself was particularly strong and that the water absorption peak of Pol c was therefore stronger. At the same time, the absorption peaks of Pol c at $1385 \mathrm{~cm}^{-1}$, $1225 \mathrm{~cm}^{-1}, 1176 \mathrm{~cm}^{-1}$ and $760 \mathrm{~cm}^{-1}$ were also obviously enhanced, which could be due to the addition of the IL component [26]. The significant difference between the infrared spectra of Pol c and Pol 0 was that a new peak appeared at $1459 \mathrm{~cm}^{-1}$, which was the absorption peak of the imidazole ring in IL. According to the above spectrum signal characteristics, it could be initially proven that IL took part in the polymerization reaction and that the expected copolymer was formed.

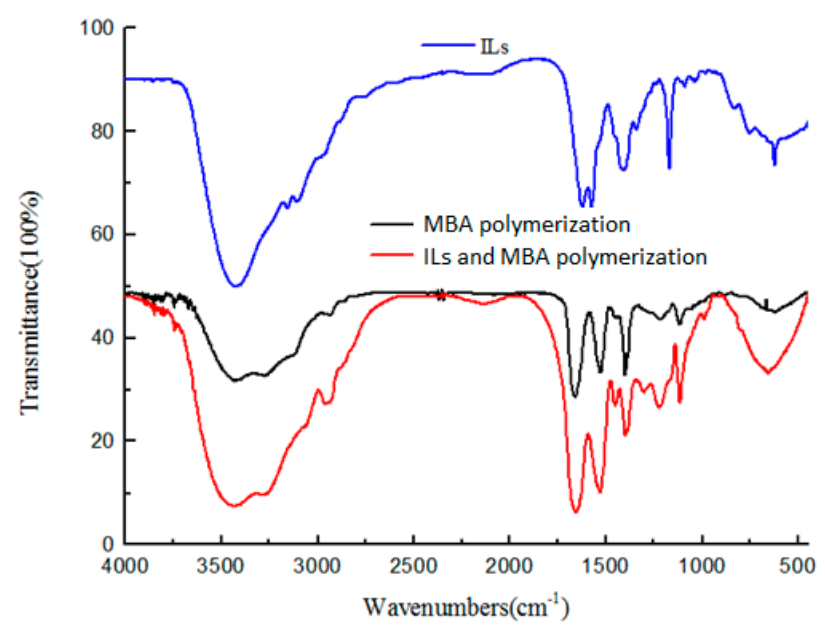

Figure 3. The IR spectrum of IL, MBA and Pol c.

\subsubsection{Elemental Analysis}

Amino acid-based ILs have a stronger polarity. No solid polymer was formed in the reaction solution with only the initiator. After MBA was added, the polymerization reaction could take place, and so MBA was an important component to realize the formation of the polymer network structure. In this experiment, ViImC ${ }_{3}$-L-Pro was employed as the polymerization monomer, and $\mathrm{K}_{2} \mathrm{~S}_{2} \mathrm{O}_{4}$ was selected as the initiator and accounted for 3\% of the total mass of the reaction raw materials. The elemental analysis results and the molar ratio of the ILs and crosslinking agent are listed in Tables 1 and 2. After the polymerization reaction was finished, the obtained polymer molecules contained IL and MBA components synchronously. Elemental analysis was used to measure the content changes of $\mathrm{C}, \mathrm{H}, \mathrm{N}$ and $\mathrm{O}$ elements in the polymers. Based on the percentage of $\mathrm{N}$ and $\mathrm{O}$ in the ILs and MBA, the content of ionic liquid in the polymer could be calculated. The yield could be obtained by changing the mass before and after the reaction.

Table 1. The elemental analysis results.

\begin{tabular}{ccccc}
\hline Polymer Monomer & $\mathbf{C}$ & $\mathbf{H}$ & $\mathbf{O}$ & $\mathbf{N}$ \\
\hline MBA & $54.5 \%$ & $6.5 \%$ & $20.8 \%$ & $18.2 \%$ \\
ViProIm+-L-Pro- & $66.6 \%$ & $4 \%$ & $12.7 \%$ & $16.7 \%$ \\
Pol c & $59.9 \%$ & $5.2 \%$ & $17.7 \%$ & $17.2 \%$ \\
\hline
\end{tabular}


Table 2. The recipe of IL Pols and their EA analysis results.

\begin{tabular}{cccc}
\hline ILs Polymer & n (IL):n (MBA) & Mass Ration of IL (\%) & Yield (\%) \\
\hline Pol 0 & $0: 1$ & 0 & 95.1 \\
Pol a & $0.25: 1$ & 15.9 & 82.3 \\
Pol b & $0.5: 1$ & 28.6 & 55.7 \\
Pol c & $0.6: 1$ & 29.9 & 50.9 \\
Pol d & $0.75: 1$ & 31.02 & 28.6 \\
Pol e & $1: 1$ & - & - \\
\hline
\end{tabular}

The contents of $\mathrm{N}$ in MBA and ViProIm+-L-Pro- molecules were $18.2 \%$ and $16.7 \%$, respectively, and the contents of O in MBA and ViProIm+-L-Pro- molecules were $20.8 \%$ and $12.7 \%$, respectively. After the polymerization reaction was finished, the obtained polymer molecules contained IL and MBA components synchronously. Elemental analysis was used to measure the content changes of $C$, $\mathrm{H}, \mathrm{N}$ and $\mathrm{O}$ elements in the polymer molecules, which was used to calculate the mass fraction of IL in the polymer molecules. With the increase of the proportion of ionic liquid IL, its mass fraction in the polymer could reach up to more than $30 \%$. On the contrary, the polymer yield decreased with the increase of the proportion of IL. When the ratio of $n(I L): n$ (MBA) was more than $0.75: 1$, the solid polymer could not be obtained, even if the amount of initiator was increased.

\subsubsection{TGA-DTG Analysis}

The product Pol c, a reaction ratio of IL: MBA of 0.6:1, was taken as an example. TG and DTG analyses were conducted to study the thermal performance of changes in the polymer mass with the temperature. The results are shown in Figure 4.

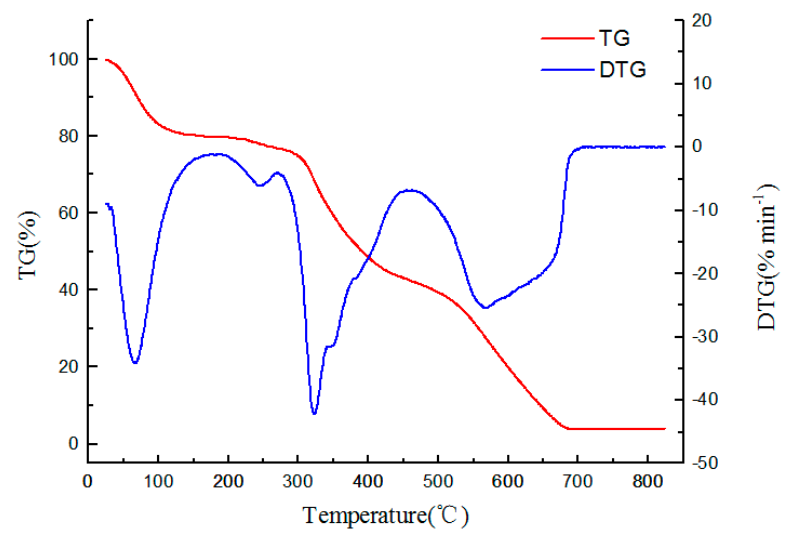

Figure 4. TGA and DTG analyses of the IL polymer.

It can be seen from the DTG curve that a mass loss below $100{ }^{\circ} \mathrm{C}$ was caused by water evaporation and accounted for about $20 \%$ of the total weight, which may be due to an incomplete drying of the polymer after synthesis. When the temperature was higher than $310^{\circ} \mathrm{C}$, the polymer began to lose weight rapidly.

It could be seen from Figure 4 that three large weight loss peaks appeared. The part under $100{ }^{\circ} \mathrm{C}$ was the reason for the loss of residual water. In addition, there was a particularly small weight loss peak at $245^{\circ} \mathrm{C}$. The weight loss peak was not completely separated from the weight loss peak at $310^{\circ} \mathrm{C}$. It could be inferred that the polymer was decomposed in three steps under the heating conditions. Moreover, the critical temperature of the two parts was relatively close around the falloff. From $200{ }^{\circ} \mathrm{C}-250{ }^{\circ} \mathrm{C}$, the amino group in the polymer began to decompose and fall off at first. At the same time, a small amount of quaternary ammonium cation degraded to form volatile components [27]. When the temperature reached $280^{\circ} \mathrm{C}$, a large weight loss peak appeared, and the degradation speed was faster. A large amount of MBA components and quaternary ammonium cation 
began to degrade [28]. When the temperature was over $480^{\circ} \mathrm{C}$, the polymer carbon skeleton started to collapse. A large weight loss peak appeared at around $540{ }^{\circ} \mathrm{C}$. Then, the weight loss rate started to slow down. After $680^{\circ} \mathrm{C}$, the weight loss reached a constant. There was very little remaining substance on the thermobalance, which might have been indicative of residual ash. The figure shows that the polymer had a great thermal stability below $60^{\circ} \mathrm{C}$.

\subsubsection{Analysis of SEM Surface Structure}

Figure 5 shows the SEM diagram of the polymer surface structure. By comparing Pol 0 and Pol c, it was found that the surface of the synthesized polymer was significantly rougher and more porous after the addition of IL liquid. When ionic liquid was added to the polymer, the surface roughness was caused by the different structure of each connecting point of the polymer. It was found that the polymers were composed of nanoscale particles. Therefore, it could be concluded that the ionic liquid polymer had a rough and porous surface and was composed of numerous nanoscale particles.

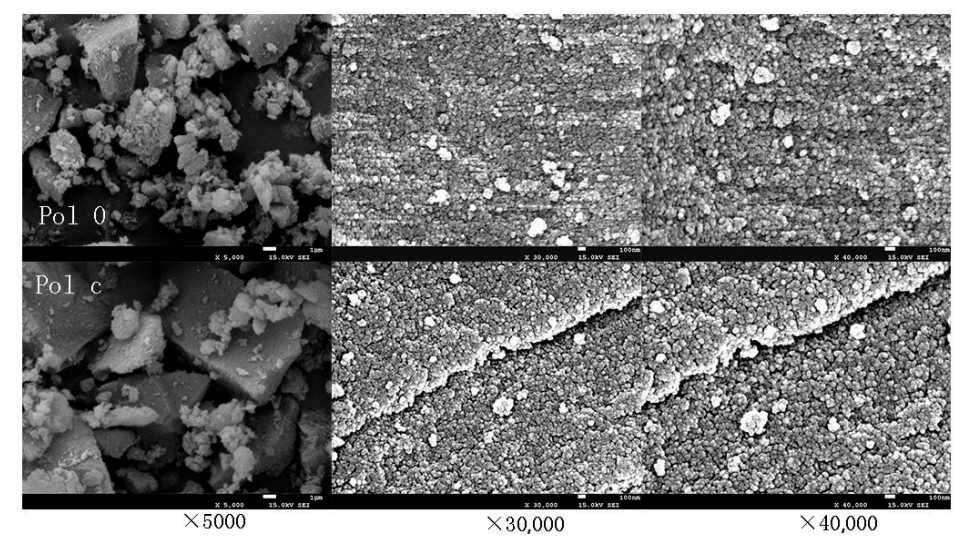

Figure 5. The SEM characterization of Pol 0 and Pol c.

\subsubsection{Particle size Distribution}

The polymer in Figure 6 was a pol c polymer. It was found that the particle size distribution of the polymer was not uniform and that the main particle size distribution was $40 \mu \mathrm{m}$ to $60 \mu \mathrm{m}$ in Figure 6 . This was mainly because the nanoparticle polymer continuously agglomerated to form a macroscopic polymer in the synthesis process. SEM research had shown that the component unit of the ionic liquid polymer was a nanometer particle, so one could see that sufficient agitation was needed in the adsorption process to break the agglomeration effect as much as possible. The smaller the particle size was, the larger the specific surface area was, which was favorable for adsorption.

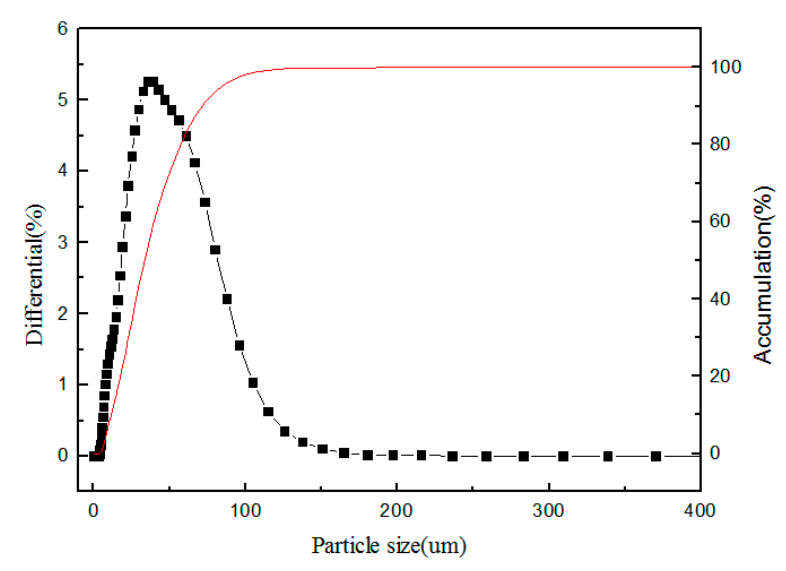

Figure 6. The particle distribution of polymers. 


\subsection{Influence of Synthesis Conditions of Ionic Liquid Polymer on Adsorption Effect}

\subsubsection{Influence of the Ratio of ILs and MBA on Adsorption}

The results of the influence of the ratio of IL and MBA in synthetic raw materials on the adsorption effect of polymer are shown in Figure 7. It could be seen from Figure 7 that the polymer formed by the polymerization of the cross-linking agent only had a poor adsorption effect on TPs, at only $60 \mathrm{mg} / \mathrm{g}$. The reason for this was that the polymer formed by MBA had a lower affinity for TPs, which resulted in a poor adsorption effect. When IL and MBA were added for polymerization, the adsorption performance of the obtained polymer was greatly improved. With the proportion of IL and MBA in the synthetic formula gradually increased, the adsorption capacity of the synthesized gel on TPs increased rapidly at first, before gradually stabilizing. When the ratio of IL to MBA continued to increase, the polymerization could not proceed. The reason for this was that the amount of cross-linking agent MBA was insufficient. Moreover, it was found that, with the increase of the ratio of IL to MBA, the yield of the polymer was also lower during the experiment. Considering the economic cost and adsorption effect, $\mathrm{n}(\mathrm{IL}): \mathrm{n}(\mathrm{MBA})=0.6: 1$ was selected as the essential condition for the next experiment.

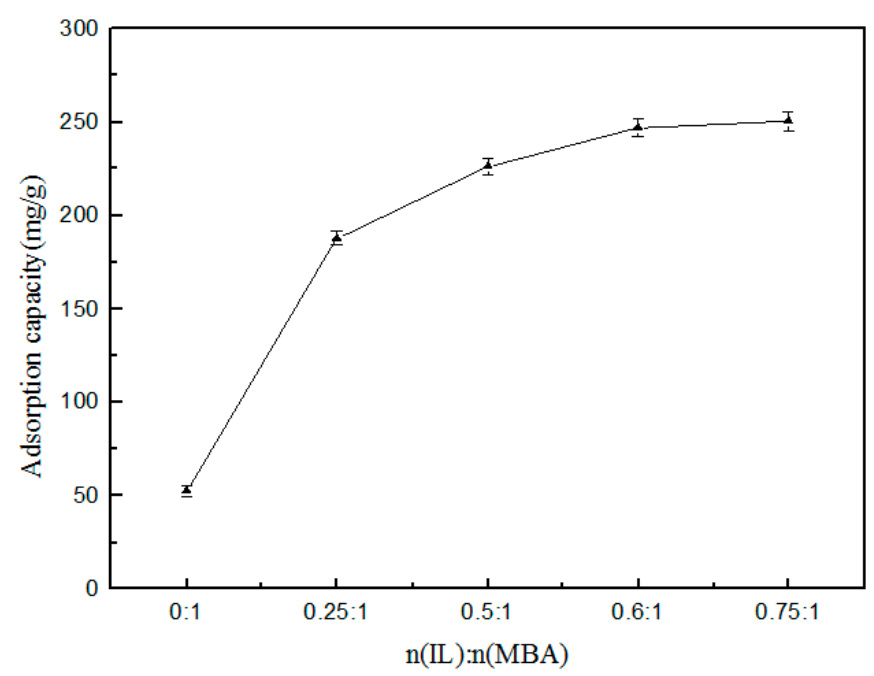

Figure 7. Effect of the amount of $n(\mathrm{IL}) / \mathrm{n}(\mathrm{MBA})$.

\subsubsection{Influence of Solvent Types on Adsorption}

The effect of the solvent type on the adsorption of TPs by the synthesized polymer is shown in Table 3. It was found that the differences of adsorption were not significant when three different solvents were used. Among them, when methanol was used as the solvent, the synthesized polymer had the best adsorption effect on TPs. When water was used as the solvent, the polymer had the second best adsorption effect. In view of green synthesis, water was suggested as a solvent, rather than methanol, although the result from methanol was somewhat better than that from water. Furthermore, if possible residuals of the solvent were to be considered, they might show a potential risk in food and pharmaceutical processes, and a water solvent would therefore be preferential.

Table 3. Effect of the types of solvents.

\begin{tabular}{ccc}
\hline Solvent & Adsorption Capacity $(\mathbf{m g} / \mathbf{g})$ & Change in Capacity (\%) \\
\hline Ethanol & 231.5 & 0 \\
Water & 239.9 & 3.6 \\
Methanol & 283.8 & 22.6 \\
\hline
\end{tabular}




\subsubsection{Influence of Ionic Liquids on Adsorption}

The adsorption of TPs by polymers formed by the polymerization of IL and MBA with different kinds of ILsis shown in Figure 8. It could be seen from Figure 8 that, when all adsorption conditions were the same, the adsorption effect of the polymer formed by double positive ILs on TPs was better than that of the polymer formed by single positive ILs. The reason for this was that when $n(I L): n(M B A)$ (dicationic ILs were half of the ratio of single positive ILs) was not changed, dicationic IL had two sites that could polymerize with the crosslinking agent, which made it easier for the chain structure to form a network polymer and convert into molecules with a complex spatial structure. The situation was beneficial to the adsorption of TPs. In addition, with the increase of the carbon chain, the unit adsorption capacity of the polymer also showed an increasing trend. Finally, (Vilm) ${ }_{2} \mathrm{C}_{6}$-(L-Pro $)_{2}$ was selected for further research.

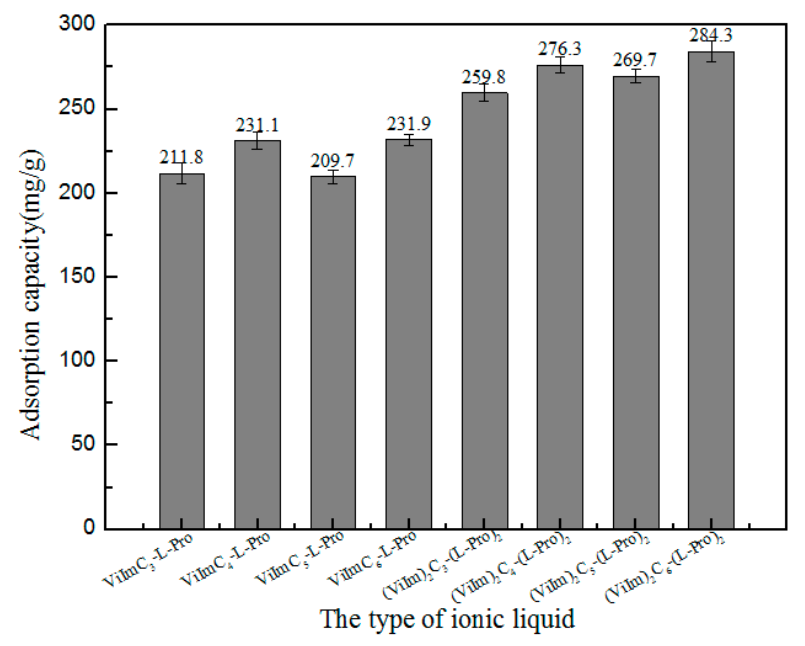

Figure 8. The types of ILs.

\subsubsection{Influence of Amount of Initiator on Adsorption}

Because the addition of the initiator $\mathrm{K}_{2} \mathrm{~S}_{2} \mathrm{O}_{4}$ was less than two percent of the total mass of the reaction raw materials, the polymerization could not occur and the polymer could not be formed; therefore, the addition of $\mathrm{K}_{2} \mathrm{~S}_{2} \mathrm{O}_{4}$ in the experiment was investigated by starting at 2.5 percent. The effect of the added amount of initiator $\mathrm{K}_{2} \mathrm{~S}_{2} \mathrm{O}_{4}$ in the formula on the adsorption amount of the formed polymer on TPs is shown in Figure 9.

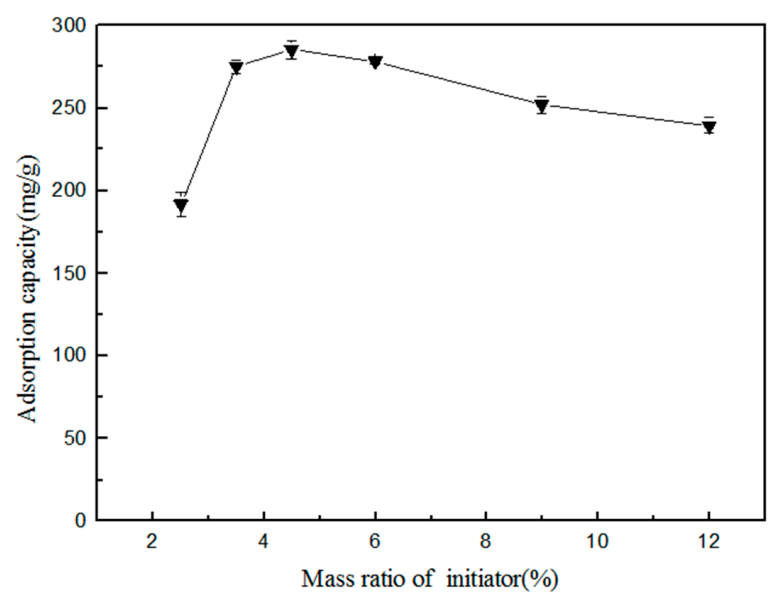

Figure 9. The mass ratio of the initiator. 
It could be seen from Figure 9 that, when the added amount of the initiator $\mathrm{K}_{2} \mathrm{~S}_{2} \mathrm{O}_{4}$ gradually increased, the adsorption amount of the synthesized polymer for TPs showed an increasing trend at first, before decreasing. Additionally, the added amount of $\mathrm{K}_{2} \mathrm{~S}_{2} \mathrm{O}_{4}$ was best around $4.5 \%$. The formed polymer had the maximum adsorption amount for TPs. In the synthetic polymers, both ILs and MBA had certain adsorption effect on TPs, but the adsorption effect of MBA was very small. The main effect of the MBA component was to form a polymer network structure to provide support. When the amount of the initiator $\mathrm{K}_{2} \mathrm{~S}_{2} \mathrm{O}_{4}$ was less than two percent, its ability to release free radicals was limited, and polymerization could not occur. When the amount of the initiator $\mathrm{K}_{2} \mathrm{~S}_{2} \mathrm{O}_{4}$ increased from $2.5 \%$ to $4.5 \%$, the free radicals released by the initiator $\mathrm{K}_{2} \mathrm{~S}_{2} \mathrm{O}_{4}$ increased, which increased the probability of ILs and MBA to obtain free radicals. The polymerization reaction occurred, and the polymer was formed. When the amount of the initiator $\mathrm{K}_{2} \mathrm{~S}_{2} \mathrm{O}_{4}$ was higher than $4.5 \%$, this was still not conducive to the full polymerization of the functional monomer and crosslinking agent, although the polymerization reaction could be completed within a very short time. Eventually, the polymerization between the monomer or crosslinking agent affected the final adsorption effect.

\subsubsection{The Performances of Adsorption and Reuse}

The results are shown in Figure 10. With the increase of the initial concentration of tea polyphenols solution, the unit adsorption capacity of the adsorbent increased and, on the contrary, the adsorption rate decreased. When the initial concentration of tea polyphenols increased from $0.5 \mathrm{mg} / \mathrm{mL}$ to $3.5 \mathrm{mg} / \mathrm{mL}$, their adsorption capacity increased from $149 \mathrm{mg} / \mathrm{g}$ to $420 \mathrm{mg} / \mathrm{g}$, and their adsorption efficiency decreased from $88 \%$ to $36 \%$.This was mainly because when the concentration was relatively low, the adsorption sites of the adsorbent were abundant and the substrates were few, which could make most of the tea polyphenols be adsorbed. However, the total amount of tea polyphenols was limited, so the adsorption amount was low. With the increase of the initial concentration, the total amount of tea polyphenols increased significantly, the adsorption sites of the adsorbent were gradually occupied, the adsorption amount increased gradually, and the adsorption rate decreased continuously.

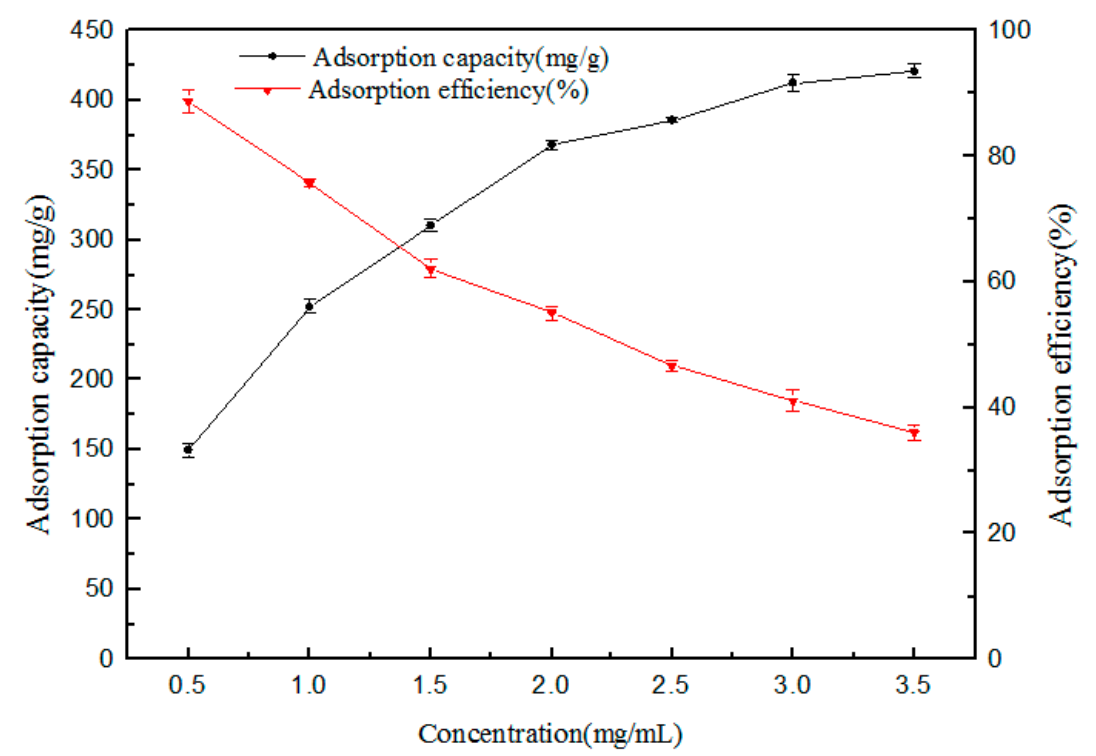

Figure 10. Effect of the initial concentration on the extraction efficiency.

The adsorption performance of TPs depended mainly on both the properties of the ionic liquid polymers and the adsorption conditions. The primary adsorption conditions, including the time, solid-liquid ratio, solution $\mathrm{pH}$ and temperature, were investigated. A typical adsorption performance obtained with the Multiple factors was $521 \mathrm{mg} / \mathrm{g}$ (synthesis conditions: solvent methanol, initiator $\mathrm{K}_{2} \mathrm{~S}_{2} \mathrm{O}_{8}$, 
ILs (ViIm) ${ }_{2} \mathrm{C}_{3}$ (L-Pro) ${ }_{2}, \mathrm{n}$ (ILs): $\mathrm{n}$ (MBA) $=0.3: 1$; adsorption conditions: solid-liquid ratio 6:2 $\mathrm{mg}: \mathrm{mL}, 45^{\circ} \mathrm{C}$, $6 \mathrm{~h}, \mathrm{pH}$ 6.0), which was greatly improved [29].

A mixed solution of TPs and theophylline was used to verify the selectivity, and the results are shown in Table 4. The extracting solution from raw material tea is usually rich in both TPs and a certain amount of theophylline, which are very similar in structure. Thus, their separation is necessary and more difficult. After adsorption, the TPs' content in the solution decreased remarkably, while the theophylline content was almost unchanged. Therefore, the selectivity was calculated at 31.85 , according to Equation (4). This indicates that the adsorption material had a very high selectivity to TPs when compared to theophylline.

Table 4. Compounds in the tea solution before and after adsorption.

\begin{tabular}{|c|c|c|c|}
\hline Adsorption & Compound & $\begin{array}{c}\text { Each Compound } \\
(\%)\end{array}$ & $\begin{array}{c}\text { Concentration of TPs or } \\
\text { Theophylline in } \\
\text { Solution }(\mathrm{mg} / \mathrm{mL})\end{array}$ \\
\hline \multirow{4}{*}{$\begin{array}{c}\text { Before } \\
\text { adsorption }\end{array}$} & ECGC & 46.4 & \multirow{3}{*}{0.40} \\
\hline & $\mathrm{EC}$ & 11.6 & \\
\hline & ECG & 4.3 & \\
\hline & Theophylline & 37.7 & 0.075 \\
\hline \multirow{4}{*}{$\begin{array}{c}\text { After } \\
\text { adsorption }\end{array}$} & ECGC & 7.5 & \multirow{3}{*}{0.15} \\
\hline & $\mathrm{EC}$ & 15 & \\
\hline & ECG & 10 & \\
\hline & Theophylline & 67.5 & 0.068 \\
\hline
\end{tabular}

The absorbed TPs on the polymer could be desorbed satisfactorily by using a $2 \%$ hydrochloric acid methanol solution as eluent. Table 5 shows the variation of the adsorption capacity after the desorption. The capacity decreased gradually over four runs. The decrease could be affected by multiple factors, such as the eluent's property, the conditions of reuses, and, particularly, its performance of adsorption. Further studies should be conducted on the performance stability and recovery conditions for practical industrial applications.

Table 5. Variations of the adsorption capacity with reuses.

\begin{tabular}{ccccc}
\hline Run Times & $\mathbf{1}$ & $\mathbf{2}$ & $\mathbf{3}$ & $\mathbf{4}$ \\
\hline adsorption capacity $(\mathrm{mg} / \mathrm{g})$ & 521 & 515 & 500 & 476 \\
\hline Variation $\%$ & 0 & -1.2 & -4.0 & -8.6 \\
\hline
\end{tabular}

\subsubsection{FTIR study on Adsorption Process}

The infrared spectrum of the polymer before and after adsorption is shown in Figure 11. It could be seen that the characteristic absorption peak of the imidazole ring $\mathrm{v}(\mathrm{C}=\mathrm{N})$ and $\mathrm{v}(\mathrm{C}=\mathrm{C})$ decreased from $1660 \mathrm{~cm}^{-1}$ to $1631 \mathrm{~cm}^{-1}$ before adsorption, which indicated that the imidazole ring of the polymer and TP was formed as a formed $\pi-\pi$ conjugate effect, with the absorption peaks showing a red-shift. In addition, it was found, by comparing the polymer adsorption before and after, that a stronger absorption peak was added at $1105 \mathrm{~cm}^{-1}$. This was the characteristic absorption peak of $\mathrm{v}(\mathrm{C}-\mathrm{O})$ of TPs [30]. 


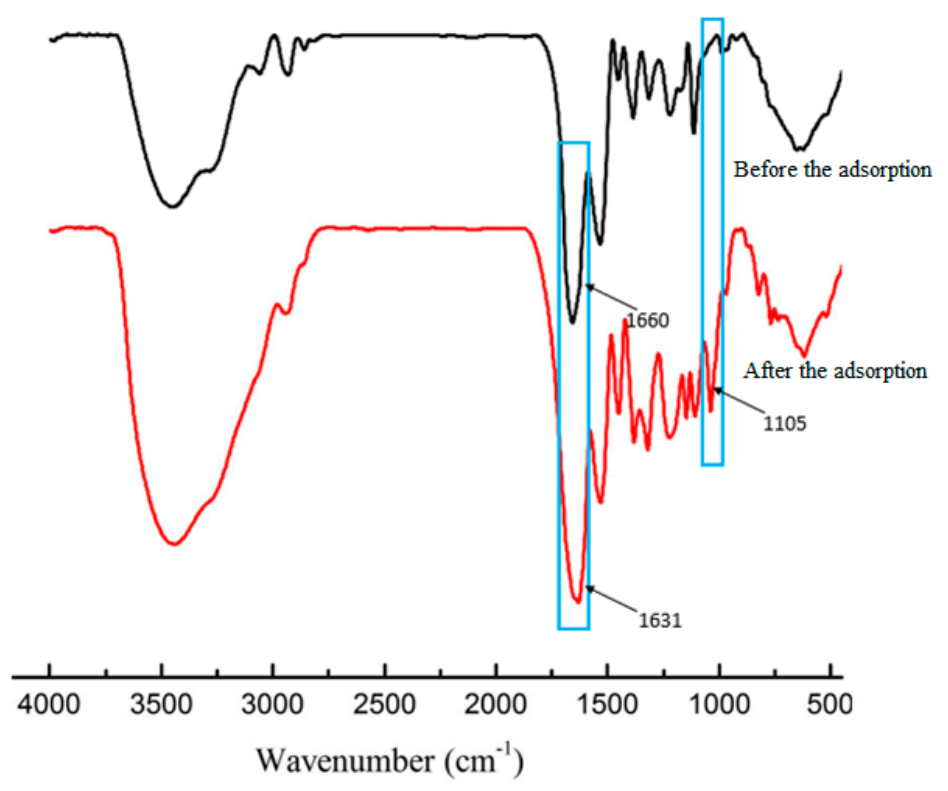

Figure 11. IR spectrum of the polymer before and after adsorption.

\section{Conclusions}

New polymer materials can be prepared by the polymerization of imidazole-type biscationic amino acid-based ILs and MBA. A typical adsorption performance obtained with this comprehensive investigation was $521 \mathrm{mg} / \mathrm{g}$ (synthesis conditions: solvent water, initiator $\mathrm{K}_{2} \mathrm{~S}_{2} \mathrm{O}_{8}$, ILs (ViIm) ${ }_{2} \mathrm{C}_{6}$ (L-Pro) ${ }_{2}$, $\mathrm{n}$ (ILs): $\mathrm{n}(\mathrm{MBA})=0.3: 1$; adsorption conditions: solid-liquid ratio $\left.6: 2 \mathrm{mg}: \mathrm{mL}, 45^{\circ} \mathrm{C}, 6 \mathrm{~h}, \mathrm{pH} 6.0\right)$, which was significantly higher than for other adsorption media.

In summary, ionic liquid polymers can be considered as a promising material for the separation of natural products, demonstrating an ideal adsorption capacity and good selectivity. They can be used in the separation of TPs from mixtures or actual samples; meanwhile, they provide a useful reference for the separation of similar components.

Supplementary Materials: The following are available online at http://www.mdpi.com/2073-4360/12/10/2171/s1.

Author Contributions: Conceptualization, H.S. and Y.L.; Formal analysis, X.H.; Investigation, F.L. and L.P.; Supervision, S.Y.; Writing-original draft, Y.L.; Writing-review \& editing, H.S. and Y.L. All authors have read and agreed to the published version of the manuscript.

Funding: This work was financially supported by the National Natural Science Foundation of China (Grant Number 81673316).

Conflicts of Interest: The authors declare that they have no known competing financial interests or personal relationships that could have appeared to influence the work reported in this paper.

\section{References}

1. Li, S.; Feng, G.; Cummings, P.T. Molecular Dynamics Study on the Capacitive Performance of Double-Cationic Ionic Liquid Electrolytes. In Proceedings of the AIChE Annual Meeting, San Francisco, CA, USA, 3-8 November 2013; p. 11.

2. Cho, W.-J.; Yeom, C.G.; Kim, B.C.; Kim, K.M.; Ko, J.M.; Yu, K.-H. Supercapacitive properties of activated carbon electrode in organic electrolytes containing single- and double-cationic liquid salts. Electrochim. Acta 2013, 89, 807-813.

3. Zhang, D.Z.; Ren, Y.Y.; Hu, Y.; Li, L.; Yan, F. Ionic liquid/poly(ionic liquid)-based semi-solid state electrolytes for lithium-ion batteries. Chin. J. Polym. Sci. 2020, 38, 506-513. [CrossRef]

4. Chen, Q.J.; An, Z.S. Synthesis of star polymeric ionic liquids and use as the stabilizers for high internal phase emulsions. Chin. J. Polym. Sci. 2017, 35, 54-65. [CrossRef] 
5. Ye, Y.; Elabd, Y.A. Relative chemical stability of imidazolium-based alkaline anion exchange polymerized ionic liquids. Macromolecules 2011, 44, 8494-8503. [CrossRef]

6. Shaplov, A.S.; Vlasov, P.S.; Lozinskaya, E.I.; Ponkratov, D.O.; Malyshkina, I.A.; Vidal, F.; Okatova, O.V.; Pavlov, G.M.; Wandrey, C.; Bhide, M.; et al. Polymeric Ionic Liquids. Comparison of Polycations and Polyanions. Macromolecules 2011, 44, 9792-9803. [CrossRef]

7. Azzaroni, O.; Brown, A.A.; Huck, W.T.S. UCST Wetting Transitions of Polyzwitterionic Brushes Driven by Self. Assoc. Angew. Chem. 2006, 118, 1802-1806. [CrossRef]

8. Wu, T.; Beyer, F.L.; Brown, R.H.; Moore, R.B.; Long, T.E. Influence of Zwitterions on Thermomechanical Properties and Morphology of Acrylic Copolymers: Implications for Electroactive Applications. Macromolecules 2011, 44, 8056-8063. [CrossRef]

9. Marcilla, R.; Ochoteco, E.; Pozo-Gonzalo, C. New Organic Dispersions of Conducting Polymers Using Polymeric Ionic Liquids as Stabilizers. Macromol. Rapid Commun. 2005, 26, 1122-1126. [CrossRef]

10. Marcilla, R.; Pozo-Gonzalo, C.; Rodríguez, J. Use of polymeric ionic liquids as stabilizers in the synthesis of polypyrrole organic dispersions. Synth. Met. 2006, 156, 1133-1138. [CrossRef]

11. Tung, T.T.; Feller, J.F.; Kim, T.Y. Electromagnetic properties of $\mathrm{Fe}_{3} \mathrm{O}_{4}$-functionalized graphene and its composites with a conducting polymer. J. Polym. Sci. Part A Polym. Chem. 2012, 50, 927-935. [CrossRef]

12. Ho, T.D.; Joshi, M.D.; Silver, M.A. Selective extraction of genotoxic impurities and structurally alerting compounds using polymeric ionic liquid sorbent coatings in solid-phase microextraction: Alkyl halides and aromatics. J. Chromatogr. A 2012, 1240, 29-44. [CrossRef] [PubMed]

13. Dr, T.F.; Kosaka, A.; Dr, Y.Y. Dramatic Effect of Dispersed Carbon Nanotubes on the Mechanical and Electroconductive Properties of Polymers Derived from Ionic Liquids. Small 2006, 2, 554.

14. Chen, X.; Li, X.; Hu, A. Advances in chiral ionic liquids derived from natural amino acids. Tetrahedron Asymmetry 2008, 19, 1-14. [CrossRef]

15. Bao, W.; Wang, Z.; Li, Y. Synthesis of Chiral Ionic Liquids from Natural Amino Acids. J. Org. Chem. 2003, 68, 591-593. [CrossRef] [PubMed]

16. Luo, S.; Xu, H.; Li, J. Facile evolution of asymmetric organocatalysts in water assisted by surfactant Brønsted acids. Tetrahedron 2007, 63, 11307-11314. [CrossRef]

17. Luo, S.; Mi, X.; Zhang, L. Functionalized ionic liquids catalyzed direct aldol reactions. Tetrahedron 2007, 63, 1923-1930. [CrossRef]

18. Ni, B.; Zhang, Q.; Headley, A.D. ChemInform Abstract Pyrrolidine-Based Chiral Pyridinium Ionic Liquids (ILs) as Recyclable and Highly Efficient Organocatalysts for the Asymmetric Michael Addition Reactions. Tetrahedron Lett. 2008, 39, 1249-1252. [CrossRef]

19. Mandal, P.; Misra, T.K.; Singh, I.D. Antioxidant activity in the extracts of two edible aroids. Indian J. Pharm. Sci. 2010, 72, 105-108.

20. Singh, B.N.; Shankar, S.; Srivastava, R.K. Green tea catechin, epigallocatechin-3-gallate (EGCG): Mechanisms, perspectives and clinical applications. Biochem. Pharmacol. 2011, 82, 1807-1821. [CrossRef]

21. Wang, L.; Gong, L.-H.; Chen, C.-J.; Han, H.-B.; Li, H.-H. Column-chromatographic extraction and separation of polyphenols, caffeine and theanine from green tea. Food Chem. 2012, 131, 1539-1545. [CrossRef]

22. Dong, W.B.; Hu, Y.; Zhang, J.H. Study on the Process of Preparation of TPs by Adsorption Resin Method. Food Sci. 2002, 23, 68-73.

23. Luo, H.M.; Xu, Y.B. Study on the adsorption properties of porous starch to TPs and the antioxidant capacity of its compounds. Tea Sci. 2015, 35, 473-480.

24. Yao, S.; Tang, J.; Song, H. Patent Application for Invention: A Method for the Extraction and Separation of Tea Polyphenols and Theine by Ionic Liquid. The National Patent CN108586458A, 28 September 2018.

25. Abdeloihid, M.; Robert, G.; Michel, H.; Deanne, L.; Svetlana, L. Spectroscopic investigation of ground state pyrrole (12C4HsN): The N-H stretch. Chem. Phys. 1997, 220, 311-322.

26. Lippert, J.L.; Robertson, J.A.; Havens, J.R.; Tan, J.S. Structural studies of poly(N-vinylimidazole) complexes by infrared and Raman spectroscopy. Macromolecules 1985, 18, 63-67. [CrossRef]

27. Juan, T.; Min, L.; Jing, G.; Hang, S. Synthesis characterization and properties of tropine-based ionic liquids gels. New J. Chem. 2019, 43, 199-207.

28. Xiao, S.; Xiao-li, S.; Yu-ming, Z.; Bei-bei, W.; Zhi-ying, Z.; Qiang, L.; Yong-hui, L. Synthesis of P123-Templated and DVB-Cross-linked Meso-macroporous Poly (ionic liquids) with High-Performance Alkylation. Appl. Organomet. Chem. 2020, 34, e5460. 
29. Zhang, W.; Feng, X.T.; Alula, Y. Bionic multi-tentacled ionic liquid-modified silica gel for adsorption and separation of polyphenols from green tea (Camellia sinensis) leaves. Food Chem. 2017, 230, 637. [CrossRef]

30. Lin, Z.Y.; Fu, J.K.; Yang, R.; Gu, P.Y. IR Absorption Spectra of Tea Polyphenol Extracted by PA/SiO 2 . J. Xiamen Univ. (Nat. Sci.) 1999, 38, 720-724. 